\title{
Coevolution between Mutualists and Parasites in Symbiotic Communities May Lead to the Evolution of Lower Virulence
}

\author{
Paul G. Nelson ${ }^{1, \star}$ and Georgiana May ${ }^{2}$ \\ 1. Ecology and Evolutionary Biology, University of Arizona, Tucson, Arizona 85721; 2. Ecology, Evolution, and Behavior, \\ University of Minnesota, St. Paul, Minnesota 55108 \\ Submitted June 28, 2016; Accepted July 24, 2017; Electronically published September 28, 2017 \\ Dryad data: http://dx.doi.org/10.5061/dryad.dd414.
}

\begin{abstract}
Most eukaryotes harbor a diverse community of parasitic, mutualistic, and commensal microbial symbionts. Although the diversity of these microbial symbiotic communities has recently drawn considerable attention, theory regarding the evolution of interactions among symbionts and with the host is still in its nascent stages. Here we evaluate the role of interactions among coinfecting symbionts in the evolution of symbiont virulence toward the host. To do so, we place the virulence-transmission trade-off into a community context and model the evolution of symbiont trophic modes along the continuum from parasitism (virulence) to mutualism (negative virulence). We establish a framework for studying multiple infections of a host by the same symbiont species and coinfection by multiple species, using a concept of shared costs, wherein the negative consequences of virulence (or harm) toward the host are shared among symbionts. Our results show that mutualism can be maintained under infection by multiple symbionts when shared costs are sufficiently low, while greater virulence and parasitism toward the host are more likely when shared costs are high. Last, for coinfection by more than one species, we show that if the presence of a mutualist ameliorates some of the costs of pathogen virulence, then the symbiotic community may more often evolve to a more commensal state and maintain mutualisms.
\end{abstract}

Keywords: coevolution, mutualism, virulence, symbiosis, trade-offs, parasitism.

\section{Introduction}

Both plant and animal eukaryotes host a diverse assembly of symbiotic species, the microbiome (Arnold et al. 2000; Qin et al. 2010). As the identity and functions of symbionts

\footnotetext{
* Corresponding author; e-mail: nels6813@umn.edu. ORCIDs: Nelson, http://orcid.org/0000-0003-4453-9895.
}

Am. Nat. 2017. Vol. 190, pp. 803-817. (C) 2017 by The University of Chicago. 0003-0147/2017/19006-57062\$15.00. All rights reserved. This work is licensed under a Creative Commons Attribution-NonCommercial 4.0 International License (CC BY-NC 4.0), which permits non-commercial reuse of the work with attribution. For commercial use, contact journalpermissions@press.uchicago.edu. DOI: $10.1086 / 694334$ in the microbiome become better understood, it is increasingly clear that few of these microbes cause disease (Dethlefsen et al. 2007; Consortium 2012). The reality of simultaneous infection by diverse symbionts in virtually all eukaryotic hosts points to a gap in our understanding of the evolution of the host-symbiont relationship, since competition among symbionts within a host is widely expected to select for increased virulence toward the host (Bremermann and Pickering 1983; Antia et al. 1994; Nowak and May 1994; Baalen and Sabelis 1995; Mosquera and Adler 1998; Gandon et al. 2002; de Roode et al. 2005; Caraco et al. 2006; Alizon et al. 2009, 2013). Consequently, the ecological factors and evolutionary processes that lead to parasitism, mutualism, or commensalism of microbes with their hosts remain an open question (Thrall et al. 2007; Rigaud et al. 2010; Afkhami et al. 2014; Lively et al. 2014). To address this gap in our understanding of symbiotic evolution, we develop an evolutionary model incorporating a trade-off between virulence and transmission to investigate whether infection by multiple symbionts necessarily causes selection for higher virulence and the breakdown of mutualism.

Parasitism and mutualism describe extremes along a continuous spectrum of symbiont relationships with the host (Johnson et al. 1997; Denison and Kiers 2004), and transitions between mutualism and parasitism may often occur (Arnold et al. 2009). However, the bodies of theory addressing evolution of mutualistic and parasitic symbiotic modes have largely developed separately and in parallel. Studies of parasitism typically explain the evolution of virulence by focusing on the dynamics of host populations, symbiont clearance, and transmission rates (Anderson and May 1979; Ewald 1980) or host resource use (Choisy and de Roode 2010). In contrast, studies of mutualism have most often used models based on game theory (Axelrod and Hamilton 1981) and focus on the conditions required for the maintenance of cooperation against cheaters, such as repeated interactions, 
host sanctions, kin selection, and mode of transmission (Doebeli and Knowlton 1998; Genkai-Kato and Yamamura 1999; West et al. 2002; Doebeli et al. 2004; Bijma and Aanen 2010; Smith et al. 2010). Consequently, we lack an understanding of causes for evolutionary transitions between mutualism and parasitism, especially in the context of the diverse symbiotic communities found in most eukaryotic hosts.

To date, most studies of symbioses have focused on pairwise interactions between host and symbiont (Stanton 2003) and invoke trade-offs for understanding the constraints on virulence (Asplen et al. 2012). For parasite-host interactions, the virulence-transmission trade-off is posited as a negative correlation between the rate of symbiont reproduction and the duration of infection in the host (Kermack and McKendrick 1932; Edmonds et al. 1975; Anderson and May 1979; May and Anderson 1983; Lenski and May 1994; Gandon et al. 2001; Alizon et al. 2009). While the virulence-transmission trade-off has been demonstrated in pathogenic interactions (Ebert and Mangin 1997; Mackinnon and Read 1999; Messenger et al. 1999; de Roode et al. 2008), a trade-off between costs to the hosts and benefits for the symbiont has been considered less often for mutualistic interactions (Herre and West 1997; Oono et al. 2011), despite its apparent explanatory power (Asplen et al. 2012). The trade-off involved in mutualism can be viewed in the same light as for parasitism; beneficial interactions with a host should result in longer infection durations but lower transmission rates (Trivers 1971; West et al. 2002; Kiers et al. 2003). In this work, we go beyond pairwise interactions of hosts and symbionts to examine the effects of interacting symbionts on the evolution of virulence toward a shared host. The results bring the transmission-virulence trade-off into the more realistic ecological context of multiple interactions within the diverse symbiont communities occupying eukaryotic hosts.

This article adds two key innovations to the existing literature. First, we allow for symbiont reproduction at no net cost to the host, which we define as a basal rate of propagule production, $b_{0}$. Doing so expands the trade-off between virulence and transmission to encompass a complete continuum from parasitism (virulence) to mutualism (negative virulence). Previous models addressing parasitism have often implicitly assumed that the value of $b_{0}$ must be 0 (Antia et al. 1994; Lenski and May 1994; Nowak and May 1994) because, by definition, parasites must harm their hosts in order to reproduce (fig. 1A). Conversely, models addressing mutualism implicitly assume that $b_{0}$ is positive because mutualists reproduce without net harm to their hosts (West et al. 2002; de Mazancourt and Schwartz 2010). In our model, when $b_{0}>$ 0 , the symbiont has the potential to evolve or maintain a mutualism (negative virulence; fig. $1 B$ ).

Second, we use a concept of shared costs to capture the effects of multiple symbionts on a shared host and then ask how shared costs affect the evolution of virulence (fig. 1C).
In contrast to previous work (Antia et al. 1994; Doebeli and Knowlton 1998; Alizon 2008), we model shared costs on a quantitative scale to capture the indirect effects of diverse symbionts on their host. The virulence-transmission tradeoff hypothesis posits that increased reproduction comes at the cost of increased harm to the host and decreased infection duration (Alizon et al. 2009). When multiple symbionts infect the same host, the virulence of one symbiont may indirectly affect the infection duration of other symbionts because of impacts on host health or mortality (West et al. 2002; de Mazancourt and Schwartz 2010); thus, costs of one symbiont's virulence may be shared among other symbionts (Alizon et al. 2013). Moreover, we can expect that not all symbionts within a host will necessarily share costs to the same degree because symbionts sharing the same host tissue may affect each other's fate to a greater degree than symbionts infecting different host tissues, especially in modular organisms such as plants. Nonetheless, the costs of virulence may also be shared incompletely among symbionts in animal hosts if they occupy differing tissues of the host, such as gut versus skin. Employing terms for both a basal rate of symbiont reproduction and shared costs of virulence, we are able to ask whether diverse interactions among members of the microbiome temper the evolution of increased virulence otherwise expected with infection by multiple symbionts (Fellous and Salvaudon 2009; Jaenike et al. 2010; Fenton et al. 2011; May et al. 2014).

\section{Methods \\ Model Description}

We take a symbiont-centered approach to the evolution of virulence. As described above, we make the classic assumption of a trade-off between the increased rate of producing transmissible propagules, $b$, at the cost of decreased infection duration due to host mortality (see fig. 1), but here, virulence indirectly affects the symbiont mortality rate $(m)$ via effects on the shared host mortality rate. Symbiont reproductive propagules have a probability of establishing a new infection, $c$, in either the current or a new host, making the rate of change in the total number of individual infections

$$
\frac{d I}{d t}=I(b c-m),
$$

where $I$ is the total number of infections, $c$ is a function of symbiont prevalence, $b$ is a function of virulence, and $m$ is a function of both the symbiont's virulence and prevalence. These functions are defined explicitly in equations (4); for a summary of terms used, see table 1 . 


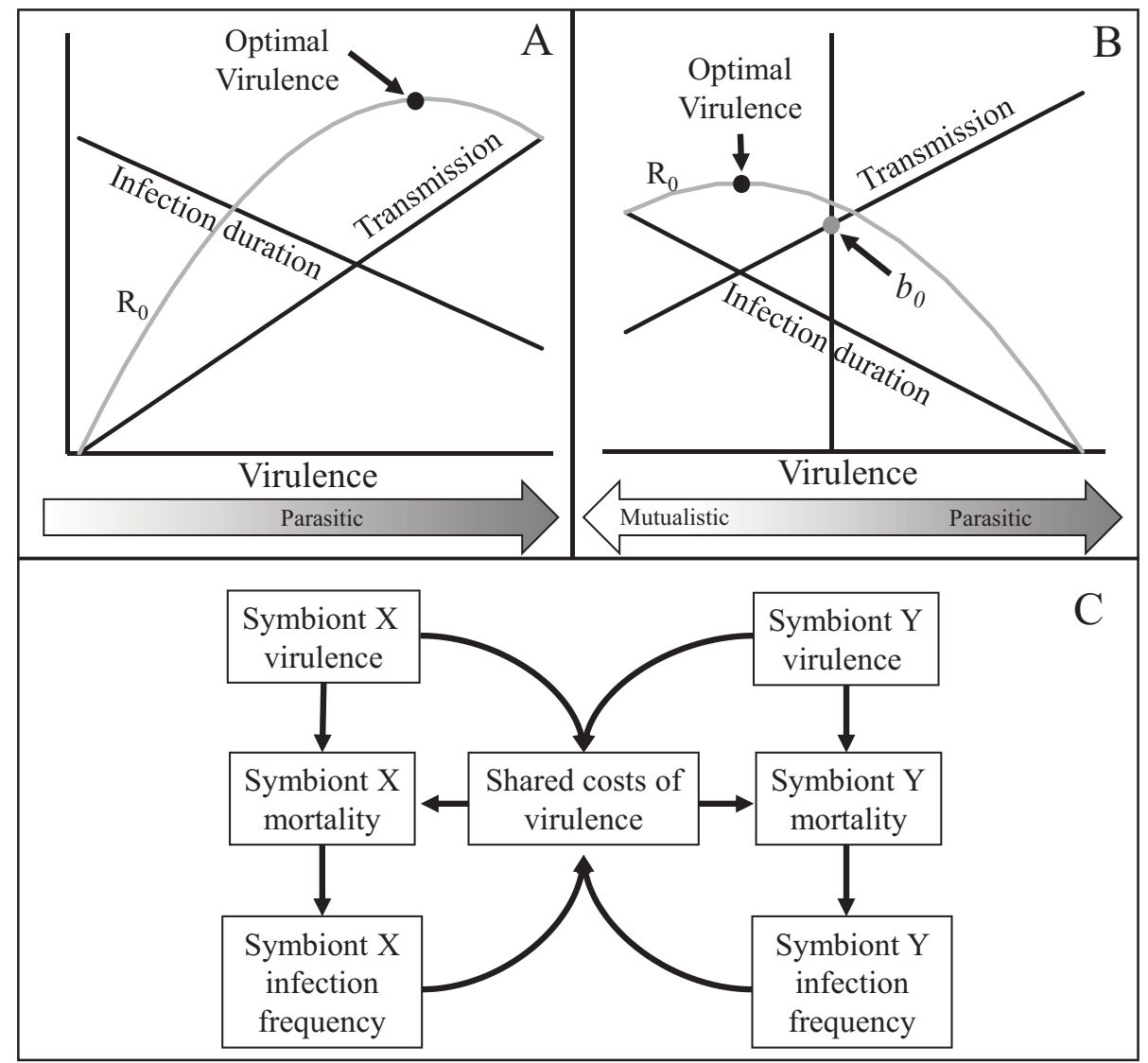

Figure 1: Model incorporates a virulence-transmission trade-off and the effects of shared costs of virulence among symbionts occupying a common host. Virulence is defined as harm to the host and is correlated with increased transmission rate at the cost of decreased infection duration. $A$, When symbionts must harm their hosts to reproduce, the symbionts are defined as having a parasitic relationship to the host. The total number of transmission events produced by a single infection $\left(R_{0}\right.$; gray) is determined by transmission rate $\times$ infection duration. Here, optimal virulence, defined as the level of virulence that maximizes the total number of transmission events over an infection's lifetime, will always be positive (black circle). $B$, Symbionts that can reproduce with no net harm to their hosts are defined as commensal or mutualistic toward the host. Here, transmission at zero virulence $\left(b_{0}\right.$; gray circle) is positive and optimal virulence can be negative, indicating a net benefit to the host. $C$, When multiple symbionts infect a host, some portion of the effects of a symbiont on the host may be shared among symbionts and thus affect each symbiont's infection duration. Consequently, the mortality rate of symbiont $\mathrm{X}$ will depend on its own virulence, the indirect effects of symbiont Y's virulence on the host, and the rate of coinfection by the two symbionts.

\section{Transmission}

We make three important assumptions regarding the probability of successfully establishing a new infection, $c$. First, the probability of establishing a new infection, $c$, is independent of the virulence. Thus, we use the term "virulence" differently than in gene-for-gene systems of plant pathogens, where virulence factors determine infectivity (Michelmore et al. 2013). Second, we assume a simple negative linear relationship between the current number of infections and the probability of establishing a new infection (Anderson and May 1979). Third, we assume that probability of infection is affected only by the number of current infections by the same symbiont species (fig. $2 A$ ).
We use the per capita rate of change of total infections $(d I / I d t)$ as a measure of symbiont fitness $w$, and we use model selection pressure on virulence as the relationship between virulence and fitness:

$$
\begin{gathered}
w=b c-m, \\
\frac{\partial}{\partial v} w=b^{\prime} c-m^{\prime} .
\end{gathered}
$$

Here $b^{\prime}$ and $m^{\prime}$ are the derivatives of each function with respect to virulence. Assuming $w$ is concave relative to virulence (where $\partial^{2} w / \partial v^{2}<0$ ), optimal virulence occurs where equation (2b) is 0 and reflects an evolutionarily stable state 
Table 1: Terms used

\begin{tabular}{ll}
\hline Term & \multicolumn{1}{c}{ Interpretation } \\
\hline$a_{b}$ & Slope of change in propagule production due to virulence \\
$a_{m}$ & Slope of change in symbiont mortality due to virulence \\
$b(v)$ & Rate of propagule production per infection \\
$b_{0}$ & Basal rate of propagule production, $b_{0}=b(0)$ \\
$c(I / N)$ & Probability of a propagule encountering and infecting a host \\
$I$ & No. infections \\
$m(v, I / N)$ & Symbiont mortality rate \\
$m_{0}$ & Basal symbiont mortality rate, $m_{0}=\sqrt{m(0, I / N)}$ \\
$N$ & No. hosts \\
$p$ & Shared costs; degree to which $m$ is affected by other symbionts within the host \\
$v$ & Virulence, increased host mortality due to infection \\
$s_{x}$ & Maximum number of infections of symbiont species $x$ per host \\
\hline
\end{tabular}

(ESS) that is refractory to invasion by genotypes that exhibit either greater or lesser virulence. Combining equation (1) with equation (2b) produces a system of ecological and evolutionary feedbacks, where every ESS (where $\partial w / \partial v=0$ ) produces an ecological equilibrium (where $d I / d t=0$ ) and every ecological equilibrium gives an ESS. When a new symbiont enters an uninfected, susceptible host population, the probability of establishing a new infection, $c$, is large, and selection on transmission is predicted to lead to evolution of a more virulent symbiont population. As the symbiont becomes more common, the probability of establishing new infections decreases. Selection pressure for rapid transmission decreases and selection to maintain current infections increases, causing the population to evolve to an intermediate level of virulence (for a detailed description, see Lenski and May 1994). The evolution of virulence is constrained such that $b$ and $m$ are positive and $c$ is between 0 and 1 . Discontinuities can arise when $\partial w / \partial v=0$ occurs outside the constraints $(b>0, I>0, m>0, c>1)$.

\section{Virulence Trade-Off}

We incorporate the trade-off between transmission and duration of infection by allowing both symbiont propagule production $(b)$ and mortality $(m)$ to increase with virulence $(v)$; that is, $\partial b / \partial v, \partial m / \partial v>0$ (figs. $1 A, 1 B, 2 C, 2 D$ ). As described above, the probability of establishing new infections $(c)$ is negatively correlated with symbiont prevalence, $\partial c / \partial(I / N)<0$ (as in Anderson and May 1979). As long as all functions are monotonic and fitness is concave relative to virulence $\left(\partial^{2} w / \partial v^{2}<0\right)$, the competitive exclusion principle holds, and each level of symbiont prevalence $(I / N)$ will yield a single, optimal virulence (Bremermann and Thieme
1989). Equations (2) reach equilibrium where $d I / I d t=0$ and $\partial w / \partial v=0$, or

$$
\frac{b^{\prime}}{b}=\frac{m^{\prime}}{m}
$$

\section{Explicit Incorporation of Shared Costs under Infection by Multiple Symbionts}

In our model, hosts may be infected by multiple genotypes of the same symbiotic species and coinfected by genotypes from multiple species (fig. $2 A$ ). Models addressing multiple symbiotic genotypes per host generally assume only two types of infecting strains and that strains on the same host share costs of virulence completely (Bremermann and Pickering 1983; Baalen and Sabelis 1995; Mosquera and Adler 1998; Friesen and Mathias 2010). Here, we use the concept of shared costs to capture the highly varied impacts of symbionts on host growth and mortality and thus the indirect and varied effects that symbionts cause to their own and other symbiont reproduction. A number of mechanisms may prevent the costs of virulence from being shared completely among symbionts. Ecological phenomena - such as spatial structure between hosts (Lipsitch et al. 1995; Caraco et al. 2006; Kerr et al. 2006) and kin structure within symbiont infections (Frank 1992) — can reduce the advantage of virulent pathogens or reinforce mutualism (Wakano et al. 2009; Harcombe et al. 2016). Additionally, host qualities — such as sanctions that specifically target virulent pathogens (Antia et al. 1994; Kiers et al. 2003) and partial tissue death or localized infections that do not harm more distant host tissues (fig. 2B) — can limit the degree to which virulent pathogens harm other symbionts within a host. We incorporate an explicit quantitative term for shared costs, $p$, to capture the level at which virulence of one symbiont affects other symbionts in that host. A singly infecting symbiont has $p=0$. A sym- 


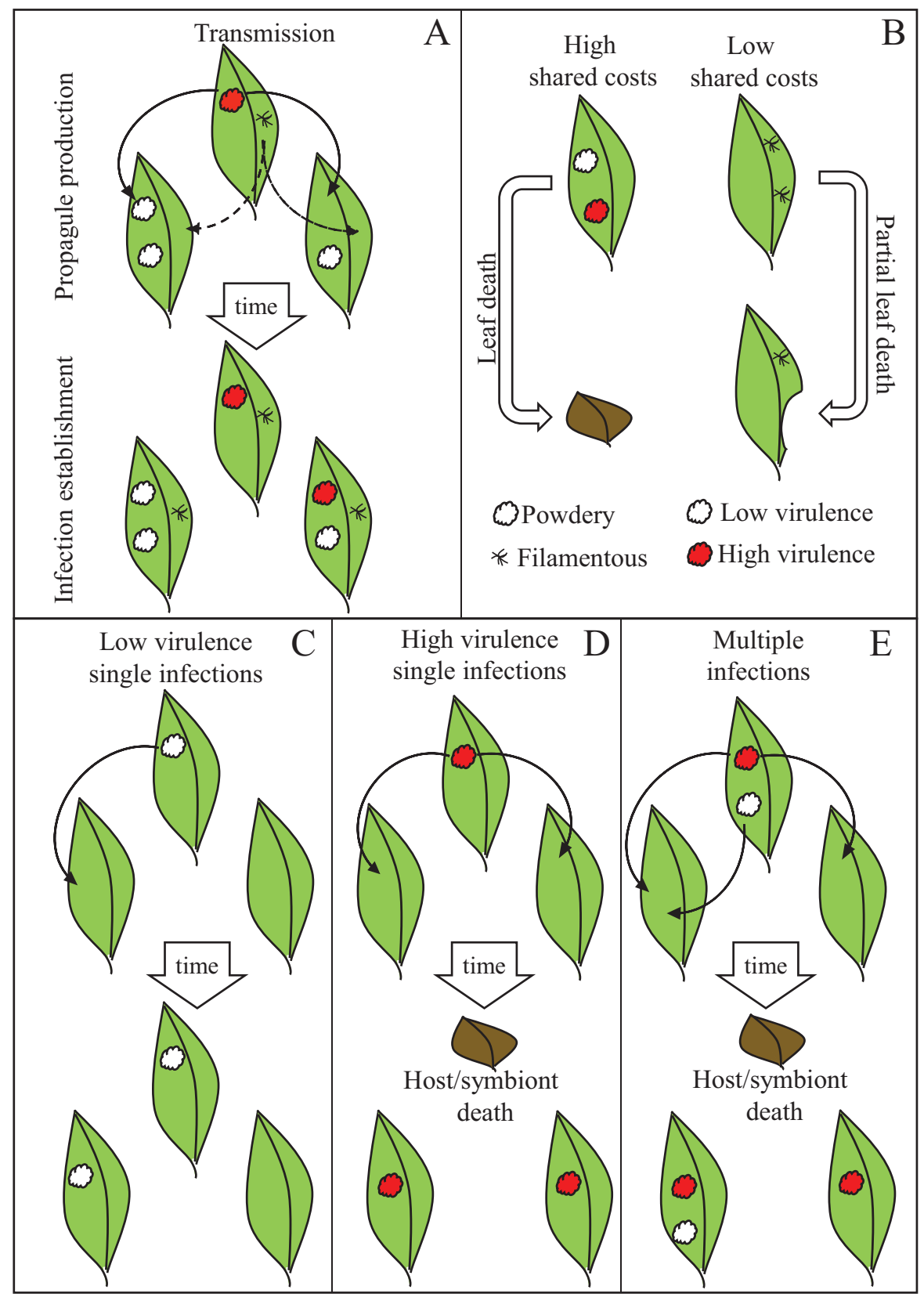

Figure 2: Model concepts. The success of symbiont transmission is dependent on the prevalence of infections of the same symbiont species. A, We consider two symbiotic species - powdery and filamentous - in a population of three hosts represented as leaves. For illustrative purposes within this figure only, we simplify density dependence to a simple rule that each host can be colonized by a maximum of two symbionts per species. Here, the left half of the leaf serves as the niche of the powdery symbionts, and the right half serves as the niche of the filamentous species. The propagules of the filamentous symbiont, indicated by dashed arrows, are unaffected by the two powdery symbionts in the leaf on the left and thus successfully infect both leaves. $B$, The degree to which symbionts affect each other's mortality is a function of shared costs. The virulent powdery symbiont (red) causes death of the entire leaf and consequently death of all other symbionts on that leaf and therefore incurs high shared costs. Virulence in the filamentous species, however, affects only surrounding host tissue, making shared costs lower. $C$, Because virulence affects both the number of propagules $(b)$ and symbiont mortality rate $(m)$, the less virulent powdery genotype (white) produces only one propagule while maintaining its current infection. $D$, In contrast, the more virulent symbiont (red) produces two propagules but - because it kills the current host - also would cause its own death and that of other symbionts in that host. In these scenarios, with single infections $(C, D)$ the fitnesses of high- and low-virulence strains are equal. $E$, However, under multiple infection by both genotypes, the more virulent genotype with higher rates of propagule production kills the shared host, putting the less virulent genotype at a competitive disadvantage. 
biont that causes only local infections may have a relatively small $p$, while a symbiont causing systemic infections may have a large $p$.

To find the total shared costs of virulence, we need to find the expected effect of each symbiont infection on other symbionts within the host. The mean virulence of symbiont species $j$ is $\bar{v}_{J}$, and the mean number of infections per host of species $j$ is $I_{j} / N$. Because we assume that virulence is additive, the total effect of coinfecting symbionts is the sum of the mean virulence of each species, adjusted by the expected frequency of encountering each species, or $\sum_{j}^{L} \bar{v}_{J} I_{j} / N$. To ensure that the absolute number of species does not itself affect total shared costs of virulence, we divide this term by the number of symbiont species $L$. Finally, the degree to which virulence of one symbiont affects other symbionts in the same host is scaled by $p$ to yield the total shared costs of virulence, $p / L \sum_{j}^{L} \bar{v}_{J} I_{j} / N$. For a nonadditive treatment of two-symbiont coevolution, see Alizon et al. (2009). The total shared costs of the virulence term accounts for the degree to which costs are shared among symbionts both within and among species $(p)$, average virulence $\left(\bar{v}_{J}\right)$, and symbiont prevalence $\left(I_{j} / N\right)$ of each symbiont species $j$ in a shared host population of size $N$.

Note that by using a weighted mean virulence for any number of $L$ symbiont species, one finds that the total shared costs of virulence do not necessarily increase with total number of species. Thus, if one were to start with a single species system and add a second species with identical parameters $\left(a_{b}, b_{0}, a_{m}, m_{0}\right)$, overall virulence and mortality rates would not change. We make this seemingly odd assumption not as an assertion of biological reality but to determine the effect of coinfection by very different symbiotic species without the total number of species as a confounding factor.

Finally, we develop explicit functions for $b, c$, and $m$. We assume that virulence yields linear reproductive benefits to symbiont species $x$ and incurs quadratic costs in mortality (Bremermann and Pickering 1983; Lenski and May 1994; Doebeli et al. 2004). Thus, for strain $k$ of species $x$,

$$
\begin{aligned}
b_{k x} & =a_{b x} v_{k x}+b_{0 x}, \\
m_{k x} & =\left[a_{m x} v_{k x}+p \frac{1}{L}\left(\sum_{j}^{L} \frac{I_{j}}{N} \bar{v}_{J}\right)+m_{0 x}\right]^{2}, \\
c_{x} & =1-\frac{I_{x}}{s_{x} N} .
\end{aligned}
$$

The term $a_{b}$ gives the reproductive payoff for virulence, and $a_{m}$ gives the penalty for virulence in symbiont mortality. Higher $a_{b}$ yields more propagules for damage done to the host, and higher $a_{m}$ increases the likelihood that an infection may die out because of harming the host. The pa- rameters $b_{0}$ and $m_{0}$ are the basal symbiont reproduction and mortality rates at zero virulence, respectively. By incorporating $b_{0}$ and $m_{0}$, a symbiont may evolve negative virulence and decrease host mortality (mutualism) at the cost of reduced symbiont reproduction, $b$. The shared costs of virulence are incorporated into the mortality rate $m$. When $p$ is 0 , equation ( $4 \mathrm{~b}$ ) expresses mortality for a single infection, and when $p>0$, equation ( $4 \mathrm{~b})$ represents the symbiont mortality rate when multiple symbionts infect the host. Note that virulence of the focal species $x$ is incorporated into both the cost of a symbiont's virulence to itself, $a_{m x} v_{k x}$, and in the shared-costs term, $p / L \sum_{j}^{L} \bar{v}_{J} I_{j} / N$, allowing the model to incorporate both multiple infections of the same species and coinfection of multiple species. Finally, $s_{x}$ is the maximum number of infections by symbiont species $x$ per host (e.g., $s=2$ in fig. 2).

We employ a negative frequency-dependent function for c (eq. [4c]) similar to Lenski and May (1994), because it is mathematically tractable and commonly used. Many mechanisms can result in frequency-dependent infection success, such as host immune response (Portugal et al. 2011), or as the result of resident symbiont functions limiting superinfection (Folimonova 2012). We assume a mass action system in which symbiont propagules are well mixed across hosts. Because the model focuses on the indirect interactions between coinfecting symbionts, we make $c$ of species $x$ a function of the infection prevalence of species $x$ and thus independent of infection prevalence of other species in the same host. This assumption that transmission success is independent of the number of infections of other species is made in order to focus on the indirect effects of shared virulence rather than the direct effect of competitive exclusion between species (as in Bronstein et al. 2003).

\section{Constraints}

Virulence is bounded such that $b$ and $m$ are nonnegative, and $I$ is bounded such that $c$ is between 0 and 1 . We make mortality rate a quadratic function of virulence following convention (Bremermann and Pickering 1983; Lenski and May 1994; Doebeli et al.2004) and for mathematical tractability. Although the model uses negative values of $v$ to represent mutualism, the quadratic function has the undesired property that highly negative values of $v$ (such that $v<-m_{0} / a_{m}$ ) instead increase mortality rates. However, for $v$ to be less than $-m_{0} / a_{m}$, symbionts must sacrifice reproduction to increase mortality rate, which would never be favored by natural selection. Thus, if $v$ is initialized such that $v<-m_{0} / a_{m}$, selection will favor less mutualistic strains until $v \geq-m_{0} / a_{m}$.

Optimal virulence is influenced by the probability of establishing a new infection $(c)$ and thus by the symbiont prevalence in the population. When new infections are readily established (fig. $3 A$ ), selection favors rapid transmission and 


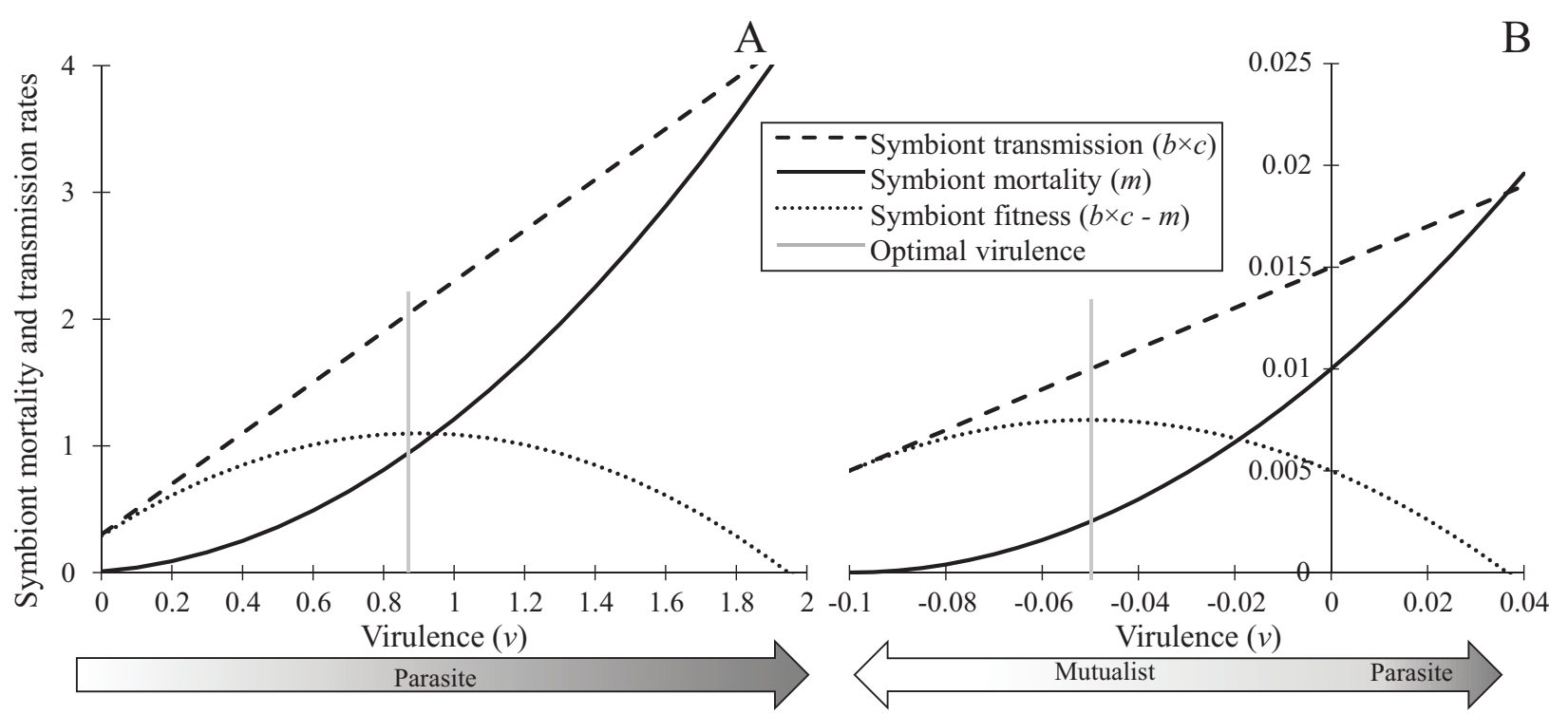

Figure 3: Optimal virulence depends on the probability of establishing a new infection, illustrated here with $c=1.0(A)$ and $c=0.05(B)$. As virulence increases, both symbiont transmission $(b \times c$; dashed line) and symbiont mortality (solid line) increase. Optimal virulence, indicated by the vertical gray lines, occurs where the symbiont per capita growth rate $(b \times c-m$; dotted line $)$ is maximized. $A$, When the probability of establishing a new infection is high $(c=1)$, selection on increased $b$ is high and the resulting optimal virulence is positive (parasitism). $B$, When the probability of establishing a new infection is low $(c=0.05)$, selection on increased $b$ is lower and the resulting optimal virulence is low, in this case negative (mutualism). In $A$ and $B, a_{b}=2, a_{m}=1, b_{0}=0.3$, and $m_{0}=0.1$. Note that $A$ and $B$ are not at ecological equilibrium, as defined by equation (3); hence, $d I / I d t>0$ when virulence is optimal.

results in positive values of optimal virulence or parasitism. When the rate of new infections is low (fig. $3 B$ ), selection favors symbionts that can persist with low mortality rates within hosts, resulting in low or even negative optimal virulence, that is, mutualism. Optimal virulence, which is an evolutionarily stable state, depends on the symbiont prevalence $(I / N)$, the value of which is determined by equation (1) and reaches an ecological stable state at $d I / d t=0$. Note that while optimal virulence away from ecological equilibrium $(d I / d t \neq 0)$ is dependent on $c$, equation (3) shows that $c$ has no effect on virulence at equilibrium for single infections. Furthermore, virulence at equilibrium is dependent only on the symbiont prevalence per host and not the absolute number of hosts, even when hosts are infected by multiple symbionts.

In contrast to the scenarios of single infections above, $c$ does affect virulence at equilibrium when hosts are infected by multiple symbionts because symbiont prevalence will affect the total shared costs of virulence $(p>0)$. For every value of $c$ and of total shared costs, $p / L \sum_{j}^{L} \bar{v}_{J} I_{j} / N$, there exists a $v_{x}^{*}=$ $\left[a_{b x} c-2 a_{m x}\left(p / L \sum_{j}^{L} \bar{v}_{J} I_{j} / N+m_{0 x}\right)\right] /\left(2 a_{m x}^{2}\right)$ for each of $L$ species such that $\partial w_{x} / \partial v_{x}=0$. If all strains within a species $x$ have virulence $v_{x}^{*}$ with corresponding fitness $w_{x}^{*}$, and a new strain with virulence $\dot{v}_{x}=v_{x}^{*}+\varepsilon$ and fitness $\dot{w}_{x}$ enters the population, the difference in growth rates between the two strains, from equation (2b), is $\dot{w}_{x}-w_{x}^{*}=-a_{m x}^{2} \varepsilon^{2}$. Because $a_{m x}$ is always positive, $\dot{w}_{x}$ is always less than $w_{x}^{*}$, making $v_{x}^{*}$ an evolutionarily stable state. This system reaches evolutionary and ecological equilibrium where $\partial w_{x} / \partial v_{x}=0, d I_{x} / d t=$ 0 , and $\overline{v_{x}}=v_{x}^{*}$ for all $L$ species, or

$$
\begin{aligned}
& v_{x}^{*}=\frac{1}{a_{m x}}\left[p \frac{1}{L}\left(\sum_{j}^{L} \frac{I_{j}}{N} \bar{v}_{J}\right)+m_{0 x}\right]-2 \frac{b_{0 x}}{a_{b x}}, \\
& \frac{I_{x}^{*}}{N}=\frac{s_{x}}{a_{b}^{2}}\left\{a_{b}^{2}+4 a_{m}^{2} b_{0}-4 a_{b} a_{m}\left[m_{0}+p \frac{1}{L}\left(\sum_{j}^{L} \frac{I_{j}}{N} \bar{v}_{J}\right)\right]\right\} .
\end{aligned}
$$

Or, of the constraint that the mortality rate must be nonnegative or $c \leq 1$ is reached,

$$
\begin{aligned}
v_{x}^{*} & =\frac{1}{a_{m x}}\left[p \frac{1}{L}\left(\sum_{j}^{L} \frac{I_{j}}{N} \bar{v}_{J}\right)+m_{0 x}\right], \\
\frac{I_{x}^{*}}{N} & =s_{x} .
\end{aligned}
$$

Note that the probability of establishing a new infection, $c$, affects only the evolved virulence through shared costs via its effect on the symbiont prevalence at equilibrium. Thus, 
while different functions for $c$ cause virulence to scale differently with $p$, evolved virulence should remain qualitatively similar to those obtained from equations (4).

\section{Estimating Parameters}

The four parameters that govern the evolution of a species' virulence under single infections $\left(a_{b}, b_{0}, a_{m}\right.$, and $\left.m_{0}\right)$ could be estimated empirically by measuring the relationship between symbiont mortality $(m)$ and rate of propagule production $(b)$ under single infections. Model parameters can thus be estimated by measuring rates of mortality and propagule production for single strain infections and fitting the equation

$$
b_{j}=\frac{a_{b}\left(\sqrt{m_{j}}-m_{0}\right)}{a_{m}}+b_{0}
$$

for each symbiont genotype, $j$. Using $b_{0}$ and $m_{0}$ estimated under single infections, $p, a_{b}$, and $a_{m}$ can be estimated by infecting hosts pairwise with two symbiont genotypes, $j$ and $k$, measuring the birth and mortality rates for each genotype $\left(b_{j k}, b_{k j}, m_{j k}\right.$, and $\left.m_{k j}\right)$ and fitting the equation

$$
a_{b}\left(\sqrt{m_{j k}}-\sqrt{m_{k j}}\right)=\left(a_{m}-p\right)\left(b_{j k}-b_{k j}\right)
$$

(for derivation, see appendix).

\section{Finding Solutions}

The equations presented here yield solutions for any set of parameters, given positive trade-off slopes and basal mortality rate $\left(a_{b}>0, a_{m}>0, m_{0}>0\right)$. For multiple infections by a single symbiont species, the total shared costs of virulence are

$$
p \frac{I^{*}}{N} v^{*}=\frac{p y-(1 / s) a_{b}^{2} a_{m} \pm \sqrt{a_{b}^{4} a_{m}^{2}-2 p a_{b}^{2} a_{m} y+p^{2}\left(a_{b}^{2}-4 a_{m}^{2} b_{0}\right)^{2}}}{8 p a_{b} a_{m}}
$$

where

$$
y=\left(a_{b}^{2}+12 a_{m}^{2} b_{0}-8 a_{b} a_{m} m_{0}\right) .
$$

Equation (6a) shows that while solutions can be defined explicitly for any number of species, closed-form solutions for more than one symbiont species are unwieldy. To efficiently generate numerical solutions, a script was written in Perl (available in the Dryad Digital Repository: http:// dx.doi.org/10.5061/dryad.dd414 [Nelson and May 2017]), which finds equilibria for $L$ species using processing time on order $L$. We find numerical solutions by testing values of shared costs $\left(p / L \sum_{j}^{L} \bar{v}_{J} I_{j} / N\right)$ for equilibria using equations (2a) and (5a). First, a test value of $p / L \sum_{j}^{L} \bar{v}_{J} I_{j} / N$ is chosen. This test value of shared costs yields a calculated $v_{x}$ for each species, according to equation (5a). Inserting the calculated $v_{x}$ and $p / L \sum_{j}^{L} \bar{v}_{J} I_{j} / N$ into equation (2a) yields a calculated $I_{x} / N$ for each species, as shown in equation (5b). Finally, the calculated shared costs are compared with the test value of shared costs and accepted as an equilibrium if within $10^{-5}$ of each other. Stability of each equilibrium is then determined by examining the sign of $d I_{x} / d t$ and $\partial w_{x} / \partial v$ at slightly higher and lower virulence and infection prevalence. In a stable equilibrium, $d I_{x} / d t$ and $\partial w_{x} / \partial v$ are both negative close to the equilibrium, while in an unstable equilibrium, $d I_{x} / d t$ and $\partial w_{x} / \partial v$ are positive on one side of an unstable equilibrium and negative on the other. In cases where the equilibrium falls outside of the constraints $(b>0, I>0$, $m>0)$, the symbiont will either become extinct or reach a state of mutualistic nonequilibrium where $m=0$, with virulence and infection frequency given by equations $(5 c)$ and (5d). For all results below, $a_{b}=4, a_{m}=1$, and $m_{0}=0.1$.

\section{Results and Discussion}

\section{Mutualism and Bistable Virulence Levels under Multiple Infections}

To determine how multiple infections per host affect the evolution of virulence, we examine the effect of increasing shared costs $(p>0)$ and use values for basal reproduction $\left(b_{0}=0.3\right)$ that yield a mutualism (negative virulence) under single infections. Our results show that mutualism can evolve and be stably maintained when shared costs are low or moderate (fig. 4 , solid line; $p<1.8$ ). We see a slight increase in virulence (or decrease in mutualism) when $p>0.2$ because of the constraint that mortality rate $(m)$ be nonnegative. As shared costs (and benefits) among mutualists increase, each mutualist must invest slightly less to maintain negligible mortality rates. Similar to the models developed by Antia et al. (1994) and Mosquera and Adler (1998), when shared costs are sufficiently high (here, $p>1.8$ ), multiple infections can lead to the evolution of increased virulence and parasitism, or a tragedy of the commons (Hardin 1968; Rankin and López-Sepulcre 2005). Notably, these results show that the outcomes of multiple or coinfections depend on the initial conditions and the identity of the symbionts, and thus a tragedy of the commons is not a necessary outcome of multiple infections.

The results show that evolved virulence can be bistable under multiple infections, with initially mutualistic populations maintaining mutualism and initially pathogenic populations evolving greater virulence. In our model, mutualists maintain high infection prevalence because of low mortality rates and thus reduce the probability of new infections establishing. In this state, pathogenic strains that depend on a relatively high probability of establishing new infections, $c$, to compensate for relatively short-lived infections may not be able to invade a population dominated by mutualistic strains 


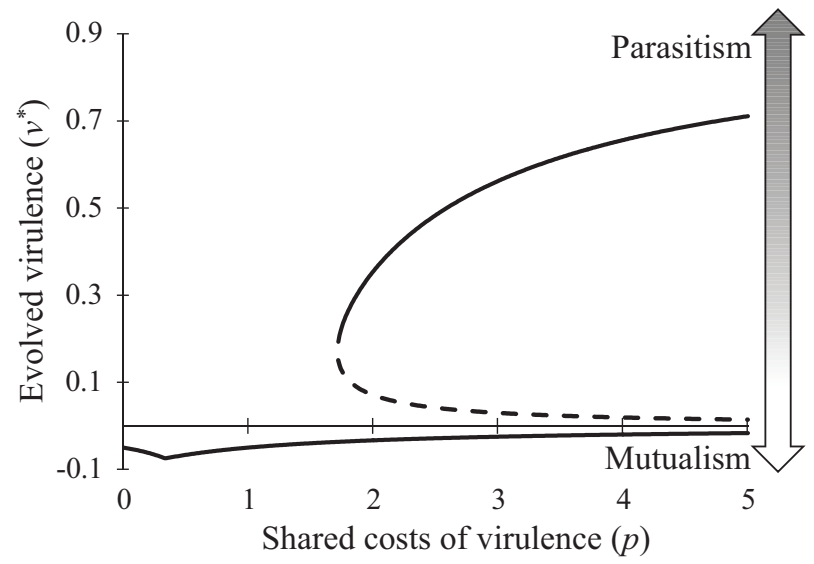

Figure 4: Evolved virulence for a potential mutualist under multiple infections. To evaluate the effects of multiple infections on the evolution of mutualism, the basal reproduction parameter is set to values under which mutualism evolves with single infections (here, $b_{0}=0.3$ ). Outcomes depend on the initial levels of virulence and $p$, the level of shared costs of virulence. Solid lines show stable equilibria, and the dashed line indicates unstable equilibria. Under conditions for which an unstable equilibrium exists, a population starting at an initial virulence level greater than that for the unstable equilibrium will evolve to a stable equilibrium of greater virulence (parasitism). In contrast, a population initially at a lower virulence than that for the unstable equilibrium will evolve to stable equilibria of negative virulence (mutualism).

of the same species. Conversely, strong pathogens kill hosts quickly, causing coinfecting mutualists to suffer decreased infection duration. Because mutualists cannot reproduce as fast as pathogens but suffer high mortality rates because of coinfecting pathogens, mutualists are unable to invade a population dominated by pathogens. The presence of unstable equilibria suggests that mutualistic but fragile communities could evolve if populations are founded by symbionts with sufficiently beneficial effects on the host.

Our finding of bistable virulence is remarkably similar to the dynamics of the continuous snowdrift game, in which actors have the option to contribute to a shared good. The snowdrift game can result in bistability of communities dominated by defectors (parasites) and cooperators (mutualists; Doebeli et al. 2004). While models of public goods have shown that bistability can arise with spatially structured populations (Wakano et al. 2009), here we show that ecological and evolutionary feedbacks can result in bistability in the absence of spatial structure. In this analysis, we have modeled the virulence trade-off as a simple quadratic function. Although more complex trade-off functions may affect the outcomes of virulence evolution (Bolker et al. 2010), the bistability between parasitism and mutualism under shared costs seems to stem from the equilibrium conditions given by equation (2). As such, our results may reflect a general feature of models with ecological and evolution feedbacks subject to similar equilibrium conditions and may be insensitive to the particular shape of the trade-off.

\section{Individual and Shared Costs Together Cause Rampant Increases in Virulence}

Results in figure 4 show virulence increasing at a decelerating rate as shared costs increase, whereas previous studies have predicted that virulence will increase at an accelerating rate as shared costs increase (Frank 1996; Alizon 2008). The difference may lie in how shared costs affect the costs of a strain's virulence to itself $\left(a_{m}\right)$ and the costs to other strains within the host $(p)$. In figure 4 , the effect of a symbiont's virulence on itself remains constant, while shared costs increase. In previous models, the costs of one strain's virulence to itself ( $a_{m}$ here, $\alpha_{m}$ in eq. [1] of Alizon [2008], and $z_{i a}$ in eq. [3] of Frank [1996]) decrease when other strains coinfect the host and the shared costs to others increase ( $p$ here, $\alpha_{r m}$ in eq. [1] of Alizon [2008], and $z_{1}$ in eq. [3] of Frank [1996]). Thus, the effects of changing the costs to the self are conflated with the effects of changing the costs of virulence to coinfecting symbionts. An advantage of our simple additive model is that we can change the cost of a symbiont's virulence to itself independently from the cost of virulence to others. To model the effects of two components of cost, we contrast the effect of changes in the individual $\left(a_{m}\right)$ and shared costs $(p)$ of virulence on the evolved symbiont mortality rate under multiple infections.

The costs of a symbiont's virulence to itself and to others play slightly different roles in the evolution of virulence among mutualists (fig. $5 \mathrm{~A}$ ) compared with among parasites (fig. $5 B$ ). Shared costs among strains of a potential mutualist lead to lower symbiont mortality, reinforcing the evolution of mutualism. Thus, virulence in a mutualist population remains negative and $m \rightarrow 0$ as the shared costs to others increase (fig. $5 \mathrm{~A}$, dotted line). In contrast, increased shared costs among pathogen strains lead to more rapidly increasing symbiont mortality, selecting for a more virulent pathogen population (fig. $5 B$, dotted line). These results show that the salient forces affecting the evolution of virulence are different for mutualists and pathogens. Within a pathogen population, evolved virulence is more strongly affected by the degree to which costs are shared between symbionts, while within a mutualist population, the individual costs more strongly affect evolved virulence. For any symbiont, when the cost of symbiont virulence to itself $\left(a_{m}\right)$ decreases as the cost to others ( $p$ ) increases (fig. 5, solid line), total mortality increases hyperbolically, resulting in runaway virulence and eventual extinction of the symbiont population. Thus, for runaway virulence to evolve, increasing shared costs must be coupled with decreasing individual costs and help explain why runaway virulence is rarely ob- 


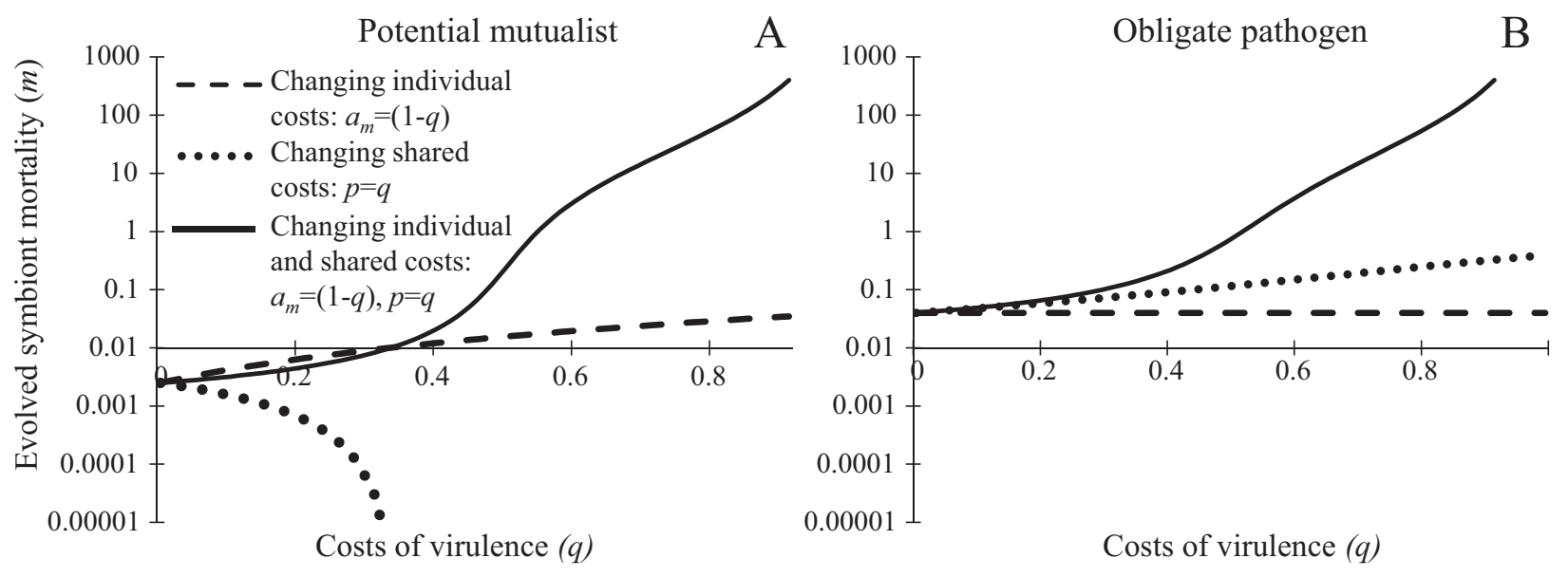

Figure 5: Dissecting the components of costs of virulence under multiple infections for a potential mutualist $\left(b_{0}=0.3 ; A\right)$ and an obligate pathogen $\left(b_{0}=0 ; B\right)$. For both, $a_{b}=4$ and $m_{0}=0.1$. Dotted lines show equilibrium symbiont mortality by shared costs $(p)$ on a log scale when the individual costs of virulence $\left(a_{m}\right)$ are constant $\left(p=q, m=\left(v+q \bar{v} I / N+m_{0}\right)^{2}\right)$. Dashed lines show evolved mortality by the individual costs of virulence, when shared costs are $0\left(a_{m}=1-q, p=0, m=\left[(1-q) v+m_{0}\right]^{2}\right)$. Finally, black lines show evolved mortality by decreasing individual costs and increasing shared costs $\left(a_{m}=1-q, p=q, m=\left[(1-q) v+q \bar{v} I / N+m_{0}\right]^{2}\right)$. Evolved symbiont mortality rates below 0.01 , denoted by the $X$-axis, indicate evolved mutualism, while rates above 0.01 indicate parasitism.

served, despite the fact that most hosts harbor multiple symbionts (Rigaud et al. 2010).

\section{Coevolution Can Lead to More Benign Symbiotic Communities}

In the complex microbiomes of most eukaryotic hosts, symbionts share their hosts with other symbionts that may have very different relationships with the host (Thrall et al. 2007; Afkhami et al. 2014). Here we investigated how coevolution between a potential mutualist and an obligate pathogen interacting within a eukaryotic host affects the evolution of virulence. Our model improves on previous efforts to study the evolution of symbiotic relationships involving multiple species (Bronstein et al. 2003; Doebeli et al. 2004) by using continuous quantitative variation for those shared costs.

To investigate the effect of shared costs between symbiont species, we contrast evolved virulence when the costs of virulence are shared both within and between a pathogen and a potential mutualist (coevolution; fig. 6A) to evolved virulence when costs are shared only within each species (evolving independently; fig. $6 \mathrm{~B}$ ). We find that when shared costs are relatively low $(p<1.5)$, coevolution between a mutualist and a parasite leads to the evolution of lower levels of virulence for both symbionts and therefore lower host and symbiont mortality. Here the mutualist becomes steadily more beneficial, and because this mitigates some of the pathogen's negative effects (fig. $6 \mathrm{~A}$, solid black line), selection for virulence in the pathogen is reduced, leading to a commensal symbiotic community with zero net virulence (solid black line along the $X$-axis for $p<1.5$ in fig. $6 C)$. In contrast, when evolving separately (fig. $6 B$ ), virulence in the mutualists remains relatively flat as shared costs increase, leading to an overall more pathogenic symbiotic community. Thus, when shared costs are low enough to support mutualism, coevolution leads to a commensal symbiotic community that is relatively robust over a range of shared costs $(0<p<1.5)$. As shared costs increase, we see a discontinuity in mean virulence - both when symbionts are coevolving and when evolved separately - corresponding to the emergence of a pathogenic equilibrium for the potential mutualist and the loss of mutualism (as shown in fig. 4). Perhaps counterintuitively, the loss of mutualism occurs at lower shared costs with coevolution (at $p \approx 1.5$ ) than without coevolution (at $p \approx 2.5$; fig. $6 C$ ). Here the erstwhile mutualist assumes a pathogenic mode that increases the pathogen's mortality rate, leading to increased overall virulence with coevolution than without. Note that coevolution between a pathogen and mutualist (fig. 6A) shows bistability, similar to a mutualist evolving alone (fig. 4); however, that bistability is present only over a relatively narrow range of shared costs $(1.5<p<2.8)$. As shared costs continue to increase $(p>2.5)$, the trajectories of mean virulence with and without coevolution converge because parasites are competing. These results show that a coevolutionary process can both stabilize commensal symbiotic communities when shared costs are low and destabilize mutualisms when shared costs are large.

That mutualistic symbionts may lead to decreased pathogen virulence suggests a testable empirical question: if mutualistic symbionts are removed from a population, do par- 

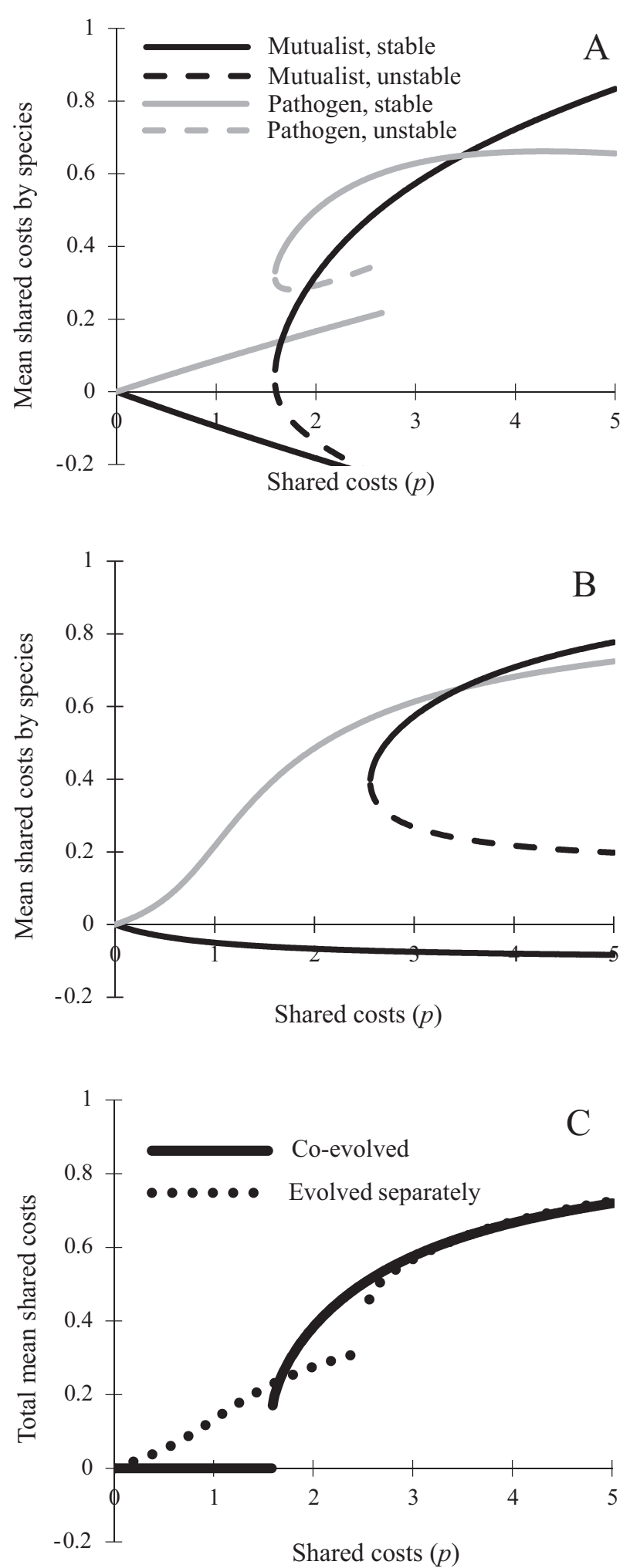

Figure 6: Total mean shared costs of two symbiotic species evolving separately and under coinfection. Mean shared costs $(p \bar{v} I / N)$ for a mutualist (black lines) and a pathogen (gray lines) are shown for each species asitic symbionts become more virulent? The results also suggest that mechanisms lowering shared costs-such as host sanctions or mutualist defense against pathogens - are likely important in the maintenance of commensal communities and to limiting the evolution of runaway virulence.

\section{Future Directions}

The model is flexible enough to incorporate additional species interactions and more complex systems of microbiomes into the additive framework. For example, the model could be extended to include host evolution, allowing examination of multihost symbionts (Woolhouse 2001; Gandon 2004) and the evolution of host resistance (Roy and Kirchner 2000; Dybdahl and Storfer 2003; Restif and Koella 2004) or tolerance (Inglese and Paul 2006). Additionally, our model includes only a single symbiont trait-virulence - and the indirect interaction of symbionts through the effects on their shared host. In reality, symbionts engage in a multifaceted array of direct and indirect interactions, and the outcomes of interactions may depend on ecological context and host quality (Bergstrom et al. 1999; Rooney and Klein 2002; Bronstein et al. 2003; Inglis et al. 2009; Jones et al. 2009; Choisy and de Roode 2010; Dyszel et al. 2010; Rodriguez Estrada et al. 2012).

Our model is intended primarily as a proof of principle (Servedio et al. 2014) to show that coinfection of differing symbionts may lead to evolution of lower virulence in a pathogen. In doing so, we illuminate parameters important to empirical systems and outline methods by which these parameters might be estimated. Our model deals with both evolutionary and ecological change; therefore, empirically testing these predictions would require the use of model organisms that are short lived and easy to manipulate (Jessup et al. 2004). Ebert and Mangin (1997) utilized Daphnia magna to test the effects of increased host mortality and, indirectly, shared costs on the evolution of virulence of the microsporidian parasite Glugoides intestinalis. The nematode Caenorhabditis elegans also provides a tractable system (Kurz and Ewbank 2000), with host and parasite generation time short enough to be amenable to studying virulence evolution. Morran et al. (2011) utilized C. elegans and the bacterial pathogen Serratia marcescens to study the host-pathogen coevolution of virulence. The greatest challenge to testing the predictions of this model regarding coevolution between symbi-

when the two species coevolve $(A)$ and when each species evolves separately $(B)$. Solid lines indicate stable equilibria, while dashed lines indicate unstable equilibria. $C$, The overall effect of coevolution on the total mean shared costs of evolved virulence $\left(p / L \sum_{j}^{L} I_{j} / N \bar{v}_{J}\right)$ is shown for coevolved symbionts (solid line) and for symbionts evolving separately (dotted line). 
onts is the dearth of model systems with multiple symbionts harbored in a common host. Thus, this model illustrates the need for further understanding and collection of model organisms' microbiota in the wild.

\section{Conclusions}

The results of our model show that the manner in which coinfecting symbionts interact and the species composition of symbiont communities within hosts are vital to explaining the diversity of symbiont interactions with their hosts. The results suggest that infection by multiple symbionts does not necessarily lead to the evolution of increased virulence and parasitism and that mutualism may be maintained if the shared costs of virulence among symbionts are limited. Moreover, because shared costs of virulence entail sharing the benefits of mutualism as well as the costs of parasitism, decreases in host mortality due to mutualists also benefit coinfecting pathogens. Thus, while coinfection by different pathogens leads to evolution of more damaging parasites, coinfection by pathogens and mutualists can result in lower virulence. Further, our results point toward a novel perspective on the many apparently commensal symbionts that make up the microbiome; rather than viewing the commensal state as a transition between the extremes of parasitism and mutualism, commensals may well be a stable state emerging from many relatively weak interactions among coinfecting microbes. Together, our results show that mutualisms may persist in the presence of multiple infections and that a diverse community of symbionts can temper rather than exacerbate the evolution of virulence.

\section{Acknowledgments}

We would like to thank Frank Shaw, Tony Dean, and Bob May for their evaluation of the equations used in this model. Additionally, we would like to thank Mike Travisano, Ruth Shaw, Eric Seabloom, and Alison Shaw for their input on the text of this manuscript. We also thank the two anonymous reviewers whose comments improved this manuscript.

\section{APPENDIX}

\section{Deriving Equations For Parameter Estimation}

For a genotype $j$ singly infecting a host, the propagule production and mortality rates are given by

$$
\begin{aligned}
m_{j} & =\left(a_{m} v_{j}+m_{0}\right)^{2}, \\
b_{j} & =a_{b} v_{j}+b_{0} .
\end{aligned}
$$

Solving each equation for virulence gives

$$
a_{b}\left(b_{j}-b_{0}\right)=v_{j}=\frac{1}{a_{m}}\left(\sqrt{m_{j}}-m_{0}\right),
$$

which reduces to

$$
\sqrt{m_{j}}=\frac{a_{m}}{a_{b}}\left(b_{j}-b_{0}\right)+m_{0} .
$$

Thus, if one can measure symbiont propagule and mortality rates for at least three symbiont genotypes with different levels of virulence, one can estimate $b_{0}, m_{0}$, and the ratio of $a_{m}$ and $a_{b}$. To estimate the shared cost of virulence $p$ and $a_{m}$ independently from $a_{b}$, one must pairwise infect hosts with two symbiont genotypes, $j$ and $k$. Assuming hosts are successfully infected by each symbiont $\left(I_{j} / N=I_{k} / N=1\right.$ for pairwise infected hosts), propagule production and mortality rates under dual infection are

$$
\begin{aligned}
m_{j k} & =\left(a_{m} v_{j}+p \overline{v_{k}}+m_{0}\right)^{2}, \\
b_{j k} & =a_{b} v_{j}+b_{0}, \\
m_{k j} & =\left(a_{m} v_{k}+p \bar{v}_{J}+m_{0}\right)^{2}, \\
b_{k j} & =a_{b} v_{k}+b_{0} .
\end{aligned}
$$

Solving for virulence of each strain and reducing, as in equations (A2), yields

$$
\begin{aligned}
& \sqrt{m_{j k}}=\frac{a_{m}}{a_{b}}\left(b_{j k}-b_{0}\right)+m_{0}+p v_{k}, \\
& \sqrt{m_{k j}}=\frac{a_{m}}{a_{b}}\left(b_{k j}-b_{0}\right)+m_{0}+p v_{j} .
\end{aligned}
$$

Next, we use the relationship between propagule production rate and virulence in equations $(\mathrm{A} 3 \mathrm{~b})$ and $(\mathrm{A} 3 \mathrm{~d})$ to remove strain virulence $v_{j}$ and $v_{k}$ :

$$
\begin{aligned}
& \sqrt{m_{k j}}=\frac{a_{m}}{a_{b}}\left(b_{k j}-b_{0}\right)+m_{0}+p \frac{1}{a_{b}}\left(b_{j k}-b_{0}\right), \\
& \sqrt{m_{j k}}=\frac{a_{m}}{a_{b}}\left(b_{j k}-b_{0}\right)+m_{0}+p \frac{1}{a_{b}}\left(b_{k j}-b_{0}\right) .
\end{aligned}
$$

Finally, subtracting equation (A5a) from equation (A5b) yields

$$
a_{b}\left(\sqrt{m_{j k}}-\sqrt{m_{k j}}\right)=\left(a_{m}-p\right)\left(b_{j k}-b_{k j}\right)
$$

which can be used to estimate the final three parameters, $a_{b}$ $a_{m}$, and $p$. 


\section{Literature Cited}

Afkhami, M. E., J. A. Rudgers, and J. J. Stachowicz. 2014. Multiple mutualist effects: conflict and synergy in multispecies mutualisms. Ecology Letters 95:833-844.

Alizon, S. 2008. Decreased overall virulence in coinfected hosts leads to the persistence of virulent parasites. American Naturalist 172:E67E79.

Alizon, S., J. C. de Roode, and Y. Michalakis. 2013. Multiple infections and the evolution of virulence. Ecology Letters 16:556-567.

Alizon, S., A. Hurford, N. Mideo, and M. Van Baalen. 2009. Virulence evolution and the trade-off hypothesis: history, current state of affairs and the future. Journal of Evolutionary Biology 22:245-259.

Anderson, R. M., and R. M. May. 1979. Population biology of infectious diseases: part I. Nature 280:361-367.

Antia, R., B. R. Levin, and R. M. May. 1994. Within-host population dynamics and the evolution and maintenance of microparasite virulence. American Naturalist 144:457-472.

Arnold, A. E., Z. Maynard, G. S. Gilbert, P. D. Coley, and T. A. Kursar. 2000. Are tropical fungal endophytes hyperdiverse? Ecology Letters 3:267-274.

Arnold, A. E., J. Miadlikowska, K. L. Higgins, S. D. Sarvate, P. Gugger, A. Way, V. Hofstetter, et al. 2009. A phylogenetic estimation of trophic transition networks for ascomycetous fungi: are lichens cradles of symbiotrophic fungal diversification? Svstematic Biology 58:283297.

Asplen, M. K., E. Bruns, A. S. David, R. F. Denison, B. Epstein, M. C. Kaiser, J. M. Kaser, et al. 2012. Do trade-offs have explanatory power for the evolution of organismal interactions? Evolution 66:1297-1307.

Axelrod, R., and W. D. Hamilton. 1981. The evolution of cooperation. Science 211:1390-1396.

Baalen, M. V., and M. W. Sabelis. 1995. The dynamics of multiple infection and the evolution of virulence. American Naturalist 146 881-910.

Bergstrom, C. T., P. McElhany, and L. A. Real. 1999. Transmission bottlenecks as determinants of virulence in rapidly evolving pathogens. Proceedings of the National Academv of Sciences of the USA 96:5095-5100.

Bijma, P., and D. K. Aanen. 2010. Assortment, Hamilton's rule and multilevel selection. Proceedings of the Roval Societv B 277:673-675; discussion, 677-678.

Bolker, B. M., A. Nanda, and D. Shah. 2010. Transient virulence of emerging pathogens. Journal of the Roval Societv Interface 7:811-822.

Bremermann, H. J., and J. Pickering. 1983. A game-theoretical model of parasite virulence. Journal of Theoretical Biology 100:411-426.

Bremermann, H. J., and H. R. Thieme. 1989. A competitive exclusion principle for pathogen virulence. Lournal of Mathematical Biology 27:179-190.

Bronstein, J. L., W. G. Wilson, and W. F. Morris. 2003. Ecological dynamics of mutualist/antagonist communities. American Naturalist 162(suppl.):S24-S39.

Caraco, T., S. Glavanakov, S. Li, W. Maniatty, and B. K. Szymanski. 2006. Spatially structured superinfection and the evolution of disease virulence. Theoretical Population Biology 69:367-384.

Choisy, M., and J. C. de Roode. 2010. Mixed infections and the evolution of virulence: effects of resource competition, parasite plasticity, and impaired host immunity. American Naturalist 175:E105E118.

Consortium, H. M. P. 2012. Structure, function and diversity of the healthy human microbiome. Nature 486:207-214. de Mazancourt, C., and M. W. Schwartz. 2010. A resource ratio theory of cooperation. Ecology Letters 13:349-359.

Denison, R. F., and E. T. Kiers. 2004. Lifestyle alternatives for rhizobia: mutualism, parasitism, and forgoing symbiosis. FEMS Microbiology Letters 237:187-193.

de Roode, J. C., R. Pansini, S. J. Cheesman, M. E. Helinski, S. Huijben, A. R. Wargo, A. S. Bell, et al. 2005. Virulence and competitive ability in genetically diverse malaria infections. Proceedings of the National Academv of Sciences of the USA 102:7624-7628.

de Roode, J. C., A. J. Yates, and S. Altizer. 2008. Virulence-transmission trade-offs and population divergence in virulence in a naturally occurring butterfly parasite. Proceedings of the National Academv of Sciences of the USA 105:7489-7494.

Dethlefsen, L., M. McFall-Ngai, and D. A. Relman. 2007. An ecological and evolutionary perspective on human-microbe mutualism and disease. Nature 449:811-818.

Doebeli, M., C. Hauert, and T. Killingback. 2004. The evolutionary origin of cooperators and defectors. Science 306:859-862.

Doebeli, M., and N. Knowlton. 1998. The evolution of interspecific mutualisms. Proceedings of the National Academv of Sciences of the USA 95:8676-8680.

Dybdahl, M. F., and A. Storfer. 2003. Parasite local adaptation: red queen versus suicide king. Trends in Ecology and Evolution 18:523-530.

Dyszel, J. L., J. N. Smith, D. E. Lucas, J. A. Soares, M. C. Swearingen, M. A. Vross, G. M. Young, et al. 2010. Salmonella enterica serovar Typhimurium can detect acyl homoserine lactone production by Yersinia enterocolitica in mice. Journal of Bacteriology 192:29-37. Ebert, D., and K. L. Mangin. 1997. The influence of host demography on the evolution of virulence of a microsporidian gut parasite. Evolution 51:1828-1837.

Edmonds, J. W., I. F. Nolan, R. C. H. Shepherd, and A. Gocs. 1975 Myxomatosis: the virulence of field strains of myxoma virus in a population of wild rabbits (Oryctolagus cuniculus L.) with high resistance to myxomatosis. Journal of Hygiene 74:417-418.

Ewald, P. W. 1980. Evolutionary biology and the treatment of signs and symptoms of infectious disease. Iournal of Theoretical Biology 86:169-176.

Fellous, S., and L. Salvaudon. 2009. How can your parasites become your allies? Trends in Parasitology 25:62-66.

Fenton, A., K. N. Johnson, J. C. Brownlie, and G. D. Hurst. 2011. Solving the Wolbachia paradox: modeling the tripartite interaction between host, Wolbachia, and a natural enemy. American Naturalist 178:333-342.

Folimonova, S. Y. 2012. Superinfection exclusion is an active viruscontrolled function that requires a specific viral protein. Journal of Virology 86:5554-5561.

Frank, S. A. 1992. A kin selection model for the evolution of virulence. Proceedings of the Roval Society B 250:195-197.

- 1996. Models of parasite virulence. Quarterly Review of Biology 71:37-78.

Friesen, M. L., and A. Mathias. 2010. Mixed infections may promote diversification of mutualistic symbionts: why are there ineffective rhizobia? Iournal of Evolutionary Biology 23:323-334.

Gandon, S. 2004. Evolution of multihost parasites. Evolution 58:455469.

Gandon, S., V. A. A. Jansen, and M. van Baalen. 2001. Host life history and the evolution of parasite virulence. Evolution 55:1056-1062.

Gandon, S., M. van Baalen, and V. A. A. Jansen. 2002. The evolution of parasite virulence, superinfection, and host resistance. American Naturalist 159:658-669. 
Genkai-Kato, M., and N. Yamamura. 1999. Evolution of mutualistic symbiosis without vertical transmission. Theoretical Population Biology 55:309-323.

Harcombe, W. R., A. Betts, J. W. Shapiro, and C. J. Marx. 2016. Adding biotic complexity alters the metabolic benefits of mutualism. Evolution 70:1871-1881.

Hardin, G. 1968. The tragedy of the commons. Science 162:1243-1248.

Herre, E. A., and S. A. West. 1997. Conflict of interest in a mutualism: documenting the elusive fig wasp-seed trade-off. Proceedings of the Royal Society B 264:1501-1507.

Inglese, S. J., and N. D. Paul. 2006. Tolerance of Senecio vulgaris to infection and disease caused by native and alien rust fungi. Phytopathology 96:718-726.

Inglis, R. F., A. Gardner, P. Cornelis, and A. Buckling. 2009. Spite and virulence in the bacterium Pseudomonas aeruginosa. Proceedings of the National Academy of Sciences of the USA 106:57035707.

Jaenike, J., R. Unckless, S. N. Cockburn, L. M. Boelio, and S. J. Perlman. 2010. Adaptation via symbiosis: recent spread of a Drosophila defensive symbiont. Science 329:212-215.

Jessup, C. M., R. Kassen, S. E. Forde, B. Kerr, A. Buckling, P. B. Rainey, and B. J. Bohannan. 2004. Big questions, small worlds: microbial model systems in ecology. Trends in Ecology and Evolution 19:189-197.

Johnson, N. C., J. H. Graham, and F. A. Smith. 1997. Functioning of mycorrhizal associations along the mutualism-parasitism continuum. New Phytologist 135:575-585.

Jones, E. I., R. Ferriere, and J. L. Bronstein. 2009. Eco-evolutionary dynamics of mutualists and exploiters. American Naturalist 174:780 794.

Kermack, W. O., and A. G. McKendrick. 1932. Contributions to the mathematical theory of epidemics. II. The problem of endemicity. Proceedings of the Roval Society A: Mathematical, Physical, and Engineering Sciences 138:55-83.

Kerr, B., C. Neuhauser, B. J. Bohannan, and A. M. Dean. 2006. Local migration promotes competitive restraint in a host-pathogen 'tragedy of the commons.' Nature 442:75-78.

Kiers, E. T., R. A. Rousseau, S. A. West, and R. F. Denison. 2003. Host sanction and the legume-rhizobium mutualism. Nature 425:78-81.

Kurz, C. L., and J. J. Ewbank 2000. Caenorhabditis elegans for the study of host-pathogen interactions. Trends in Microbiology 8:142144.

Lenski, R. E., and R. M. May. 1994. The evolution of virulence in parasites and pathogens: reconciliation between two competing hypotheses. Journal of Theoretical Biology 169:253-265.

Lipsitch, M., E. A. Herre, and M. A. Nowak. 1995. Host population structure and the evolution of virulence: a "law of diminishing returns." Evolution 49:743-748.

Lively, C. M., J. C. de Roode, M. A. Duffy, A. L. Graham, and B. Koskella. 2014. Interesting open questions in disease ecology and evolution. American Naturalist 184(suppl.):S1-S8.

Mackinnon, M. J., and A. F. Read. 1999. Genetic relationships between parasite virulence and transmission in the rodent malaria Plasmodium chabaudi. Evolution 53:689-703.

May, G., P. Nelson, and K. Clay. 2014. Defensive mutualisms: do microbial interactions within hosts drive the evolution of defensive traits? Functional Ecology 28:356-363.

May, R. M., and R. M. Anderson. 1983. Epidemiology and genetics in the coevolution of parasites and hosts. Proceedings of the Roval Society B 219:281-313.
Messenger, S. L., I. J. Molineux, and J. J. Bull. 1999. Virulence evolution in a virus obeys a trade-off. Proceedings of the Roval Society B 266:397-404.

Michelmore, R. W., M. Christopoulou, and K. S. Caldwell. 2013 Impacts of resistance gene genetics, function, and evolution on a durable future. Annual Review of Phytopathology 51:291-319.

Morran, L. T., O. G. Schmidt, I. A. Gelarden, R. C. I. Parrish, and C. M. Lively 2011. Running with the Red Queen: host-parasite coevolution selects for biparental sex. Science 333:216-218.

Mosquera, J., and F. R. Adler. 1998. Evolution of virulence: a unified framework for coinfection and superinfection. Journal of Theoretical Biology 195:293-313.

Nelson, P., and G. May. 2017. Data from: Coevolution between mutualists and parasites in symbiotic communities may lead to the evolution of lower virulence. American Naturalist, Dryad Digital Repository, http://dx.doi.org/10.5061/dryad.dd414.

Nowak, M. A., and R. M. May. 1994. Superinfection and the evolution of parasite virulence. Proceedings of the Roval Societv B 255:81-89.

Oono, R., C. G. Anderson, and R. F. Denison. 2011. Failure to fix nitrogen by non-reproductive symbiotic rhizobia triggers host sanctions that reduce fitness of their reproductive clonemates. Proceedings of the Royal Society B 278:2698-2703.

Portugal, S., C. Carret, M. Recker, A. E. Armitage, L. A. Goncalves, S. Epiphanio, D. Sullivan, et al. 2011. Host-mediated regulation of superinfection in malaria. Nature Medicine 17:732-737.

Qin, J., R. Li, J. Raes, M. Arumugam, K. S. Burgdorf, C. Manichanh, T. Nielsen, et al. 2010. A human gut microbial gene catalogue established by metagenomic sequencing. Nature 464:59-65.

Rankin, D. J., and A. López-Sepulcre. 2005. Can adaptation lead to extinction? Oikos 111:616-619.

Restif, O., and J. Koella. 2004. Evolution of resistance and tolerance to pathogens. American Naturalist 164:E90-E102.

Rigaud, T., M. J. Perrot-Minnot, and M. J. Brown. 2010. Parasite and host assemblages: embracing the reality will improve our knowledge of parasite transmission and virulence. Proceedings of the Roval Society B 277:3693-3702.

Rodriguez Estrada, A. E., W. Jonkers, H. C. Kistler, and G. May 2012. Interactions between Fusarium verticillioides, Ustilago maydis, and Zea mays: an endophyte, a pathogen, and their shared plant host. Fungal Genetics and Biology 49:578-587.

Rooney, P. J., and B. S. Klein. 2002. Linking fungal morphogenesis with virulence. Cellular Microbiology 4:127-137.

Roy, B. A., and J. W. Kirchner. 2000. Evolutionary dynamics of pathogen resistance and tolerance. Evolution 54:51-63.

Servedio, M. R., Y. Brandvain, S. Dhole, C. L. Fitzpatrick, E. E. Goldberg, C. A. Stern, J. V. Cleve, et al. 2014. Not just a theory-the utility of mathematical models in evolutionary biology. PLoS Biology 12:1-5.

Smith, J., J. D. Van Dyken, and P. C. Zee. 2010. A generalization of Hamilton's rule for the evolution of microbial cooperation. Science 328:1700-1703.

Stanton, Maureen L. 2003. Interacting guilds: moving beyond the pairwise perspective on mutualisms American Naturalist 162 (suppl.):S10-S23.

Thrall, P. H., M. E. Hochberg, J. J. Burdon, and J. D. Bever. 2007. Coevolution of symbiotic mutualists and parasites in a community context. Trends in Ecology and Evolution 22:120-126.

Trivers, R. L. 1971. The evolution of reciprocal altruism. Quarterly Review of Biology 46:35-57. 
Wakano, J. Y., M. A. Nowak, and C. Hauert. 2009. Spatial dynamics of ecological public goods. Proceedings of the National Academy of Sciences of the USA 106:7910-7914.

West, S. A., E. T. Kiers, E. L. Simms, and R. F. Denison. 2002. Sanctions and mutualism stability: why do rhizobia fix nitrogen? Proceedings of the Roval Society B 269:685-694.
Woolhouse, M. E. J. 2001. Population biology of multihost pathogens. Science 292:1109-1112.

Associate Editor: Benjamin M. Bolker Editor: Yannis Michalakis
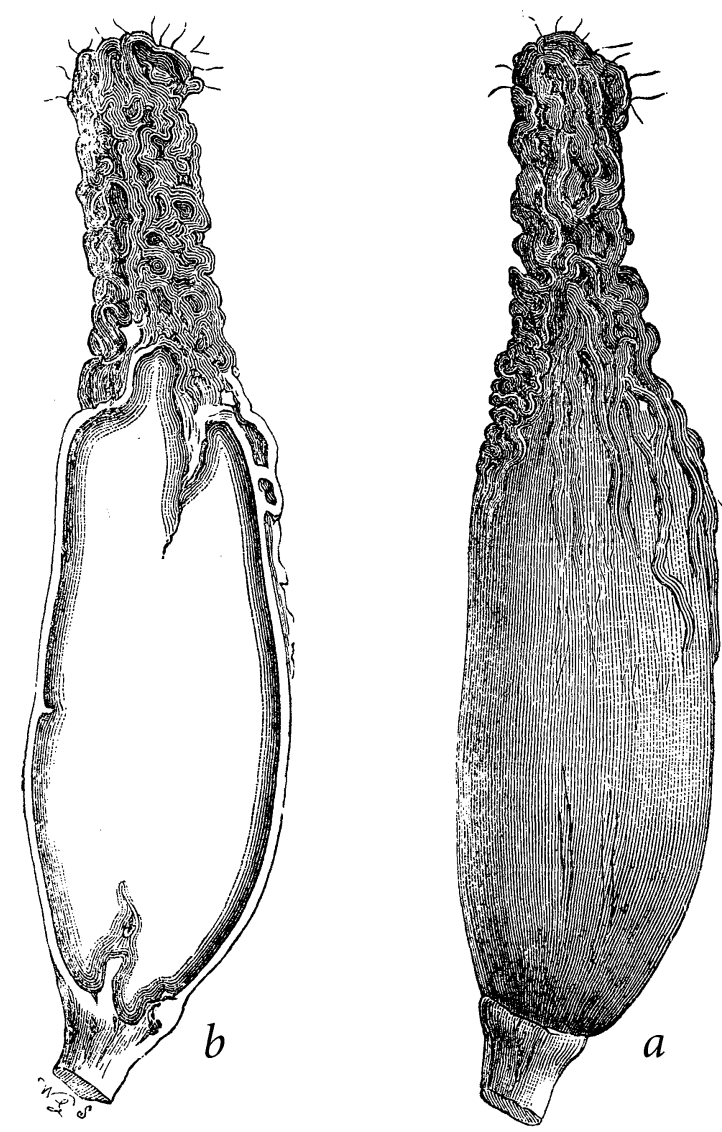

"Ergotted ear of Rye, showing the early sphacelia state at the apex, and the young Ergot at the base. $a$, external appearance; $b$, section." From William Carruthers, "On Ergot" (The American Naturalist, 1875, 9:450-465). 